\title{
Effects of Children on Divorce Probabilities and of Divorce on Fertility: The Case of Finland $1984^{1}$
}

\section{WOLFGANG LUTZ}

\author{
Leader, Docent \\ Population Projects \\ IIASA \\ Laxenburg, Austria
}

\begin{abstract}
As a follow-up to the article, "The Demographic Dimensions of Divorce: The Case of Finland" by W. Lutz, A.B. Wils, and M. Nieminen, in Population Studies, 1991, this study looks explicitly at the interactions between childbearing and divorce. Specifically, the study looks at the effects of parity and age of the youngest child on divorce probabilities controlling duration of marriage, and the effect of marital status and the duration since divorce on parity-specific birth probabilities. Generally, controlling for other demographic covariates tends to change the usually considered bivariate associations between childbearing and divorce. For instance, when considering marital duration divorce probabilities are highest for childless women and lowest for women with two or three children, whereas the bivariate perspective shows a peak for parity one women.
\end{abstract}

Keywords: divorce, fertility, parity, Finland

\section{Introduction}

This paper looks at the strictly demographic covariates of divorce and fertility. In particular it looks at the way divorce probabilities vary with the number of children and with the age of the youngest child and, on the other hand, at the influence of divorce on childbearing behavior controlling for parity and time since divorce.

In a way this short study is an extension of the paper "The Demographic Dimensions of Divorce: The Case of Finland" by W. Lutz, A.B. Wils, and M. Nieminen in Population Studies 1991. In that paper we attempted an assessment of the relative effects of duration of marriage, period, and cohort for historical divorce trends in Fin-

${ }^{1}$ Paper presented at Session 19 at the European Population Conference, Paris, October 21-25, 1991. The research assistance of Anne B. Wils in the preparation of this paper is gratefully acknowledged. 
land and of age, age at marriage and duration of marriage for Finnish period data of 1984. In the first case the period effect turned out to be dominating; in the second case age proves to be the most important factor in explaining the high divorce rates of young women with a young age at marriage and marital durations $3-5$. That analysis used multivariate logit models of the age-period-cohort (APC) type to assess the relative effects of the various demographic dimensions of divorce.

The present paper is also based on the above-mentioned data set from the Finnish population register. This data set covers the complete population of Finland in 1984 (4.8 million individuals). It cross-classifies the total population as of 1 January 1984 by sex, age, marital status, duration in marital status, order of marriage, number of children, and age of youngest child. This results in a very large number of cells, for which the number of persons in the cell, and the numbers of births, marriages, divorces, deaths of spouses, and own deaths over the course of 1984 are given. Special counts are given for some more likely multiple events such as marriage and birth in the same year. A large number of demographic probabilities may be derived from this unique data set.

Since the dimensions of individual time (age, duration in status, age of youngest child) are measured in completed years at the very beginning of 1984 , the exact age or duration at the occurrence of an event during 1984 may be of up to two years greater than the stated age or duration. For example the exact age of women in the age category 25 who experience a birth during 1984 may range from 25 plus one day (25th birthday on 31 December 1983, birth on 1 January 1984) to 27 minus one day (25th birthday on 1 January 1983, birth on 31 December 1984). This systematic upward bias by about one year on average applies to all durations and ages stated and needs to be kept in mind when interpreting the results. Especially at very low durations such as divorces at duration zero the results look more plausible if one considers the fact that these women are on average already married one year.

The empirical figures given in the following are called probabilities and not rates because they relate the events to an initial stock of persons exposed to the risk of the event at the beginning of the year and do not consider the person years of exposure as rates would do. The probabilities in sections 2 and 3 are derived from multivariate logit regressions in which each age and duration category are represented by dummy variables $^{2}$. This provides estimates for average age and duration patterns that may then be combined with additional effects. In sections 4 and 5 empirically-measured probabilities are given. These more direct measures can be used because there we do not disaggregate as much as in sections 2 and 3, and they were preferable because they require less assumptions.

\section{The effect of parity on divorce probabilities}

Figure 1 gives divorce probabilities in Finland in 1984 by parity for selected marital durations and for all durations together. The curve for all durations together is distinctly different from those which control for duration of marriage. For all married women together the annual divorce probability is around $1.2 \%$ in the case of parity zero, increases to $1.3 \%$ for women with one child and declines thereafter. For women with three or four children the probability is only $0.7 \%$. Hence, this bivariate analysis of divorce and parity gives the impression that divorce risks are highest for women with one child.

\footnotetext{
${ }^{2}$ For a more detailed description of the method see Hobcraft and Casterline, 1983, and Lutz, 1989.
} 
When looking at different categories of marital duration separately, divorce probabilities are highest for childless women. They tend to decline slightly to parity 1 and somewhat more to parities 2 and 3 . For parities 4 and above again a slight increase is observed. Hence these duration-specific curves give a U-shaped pattern with respect to parity. The curve for duration zero is lowest, for duration 5 highest, and for durations 10 and 20 in between. This corresponds to the observed variation of divorce rates by marital duration which steeply increase up to duration 5 and slowly decline thereafter.

The reason for the discrepancy between the duration-specific curves and all durations is the changing weights at different durations of marriage of women of different parities. Women at parity zero include a large group of newlyweds who have low divorce risks. The peak at parity one that appears for all durations taken together can then be explained by the large proportion of women in the highest risk durations (3-6 years) who have one child. The further decrease with higher parities also reflects the lower divorce probabilities of older women with higher marital durations who have more weight in those parity categories. In summary we can say that the duration effect dominates the parity effect, so that the latter becomes only visible once we control for duration.

It is also interesting to look at the difference between men and women in the way the number of children affects divorce probabilities. To make the differences more clearly visible the cumulated probabilities up to duration 20 of not divorcing ("surviving" in marriage) disregarding mortality were calculated for men and women of parities zero and one. Since the given data cannot follow the maternity and paternity history

Fig u re 1. Probabilities of divorce by parity for selected marital durations: Finnish women in 1984. (Results of a multivariate logit model considering parity and duration of marriage.)

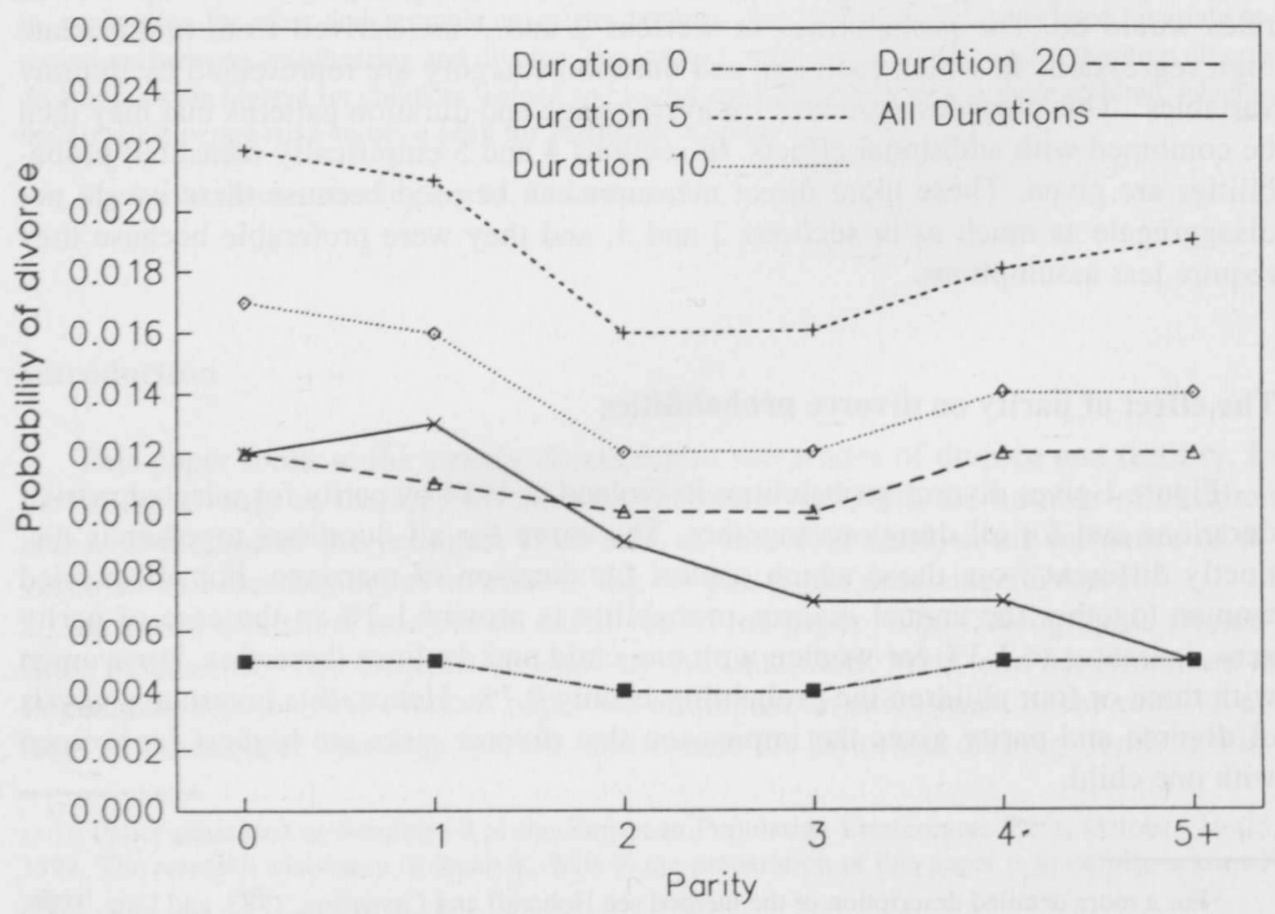


$\mathrm{T}$ a b le 1. Cumulated probabilities of remaining married for men and women with no children or one child, based on Finnish period data for 1984.

\begin{tabular}{ccccc} 
& \multicolumn{2}{c}{ No children } & \multicolumn{2}{c}{1 child } \\
Marital duration & Men & Women & Men & Women \\
5 & 0.876 & 0.883 & 0.921 & 0.914 \\
10 & 0.775 & 0.782 & 0.831 & 0.818 \\
15 & 0.717 & 0.727 & 0.767 & 0.754 \\
20 & 0.680 & 0.693 & 0.720 & 0.708
\end{tabular}

of individuals the figures are derived from cumulating period probabilities by parity. Since usually parity status changes over the marital history, this measure is only unproblematic in the case of parity zero. But also for parity one it can be considered a useful approximation because generally first children are not born at high marital durations (Table 1).

Childless men tend to have slightly higher divorce probabilities than childless women. By duration 20 the figures indicate $32.0 \%$ of all childless married men divorced while the figure for women is only $30.7 \%$. Since in this case own children are considered, discrepancies between men and women are due to children with previous partners. For men with one child the probability of having divorced after 20 years of marriage is only $28.0 \%$ as opposed to $29.2 \%$ for women. Put differently, the fact of having fathered a child reduces the probability of a divorce after 20 years of marriage by 4 percentage points, whereas for women the birth of a child results only in a reduction of 1.5 percentage points. Hence, if no other unconsidered factors determine this pattern, one could say that the higher marital stability associated with a child is more pronounced for men than for women.

\section{Age of youngest child and divorce}

Since our data set also has information on the age of the youngest child, it is interesting to look how divorce probabilities are affected by that age. Again we attempt to measure the unconfounded effect which is assumed to be found after controlling for marital duration. Figure 2 gives the variation of divorce intensities of women by the age of the youngest child for selected marital durations and for all durations together.

All curves show a minimum of divorce probabilities at age 1 which actually means that the youngest child is on average around age 2 according to the definitions given in the introduction. The higher value at age zero is distorted by the fact that childless couples had been assigned that value. After age 1 all curves steeply increase up to age 4. After that the curve for all durations together levels off and even slowly declines to the highest age categories, whereas the duration-specific curves continue to increase. It is noteworthy that divorce probabilities are especially high if duration of marriage is less than age of the youngest child, i.e. if the youngest child was born before the current marriage was contracted. The levelling off of the aggregate curve above age 4 may again be explained by the changing weights, i.e. higher ages and marital durations with higher ages of the youngest child.

The most remarkable finding of this is that even when controlling for duration there seems to be a strong positive association between age of youngest child and divorce risk between ages 1 and 4 . The extent of this effect becomes even somewhat stronger when controlling for duration. Divorce risks with a child around age 5 are about three 
F i g u re 2. Estimated divorce probabilities by age of the youngest child for selected marital durations, Finland, 1984. (Results of a multivariate logit model considering age of youngest child and duration of marriage.)

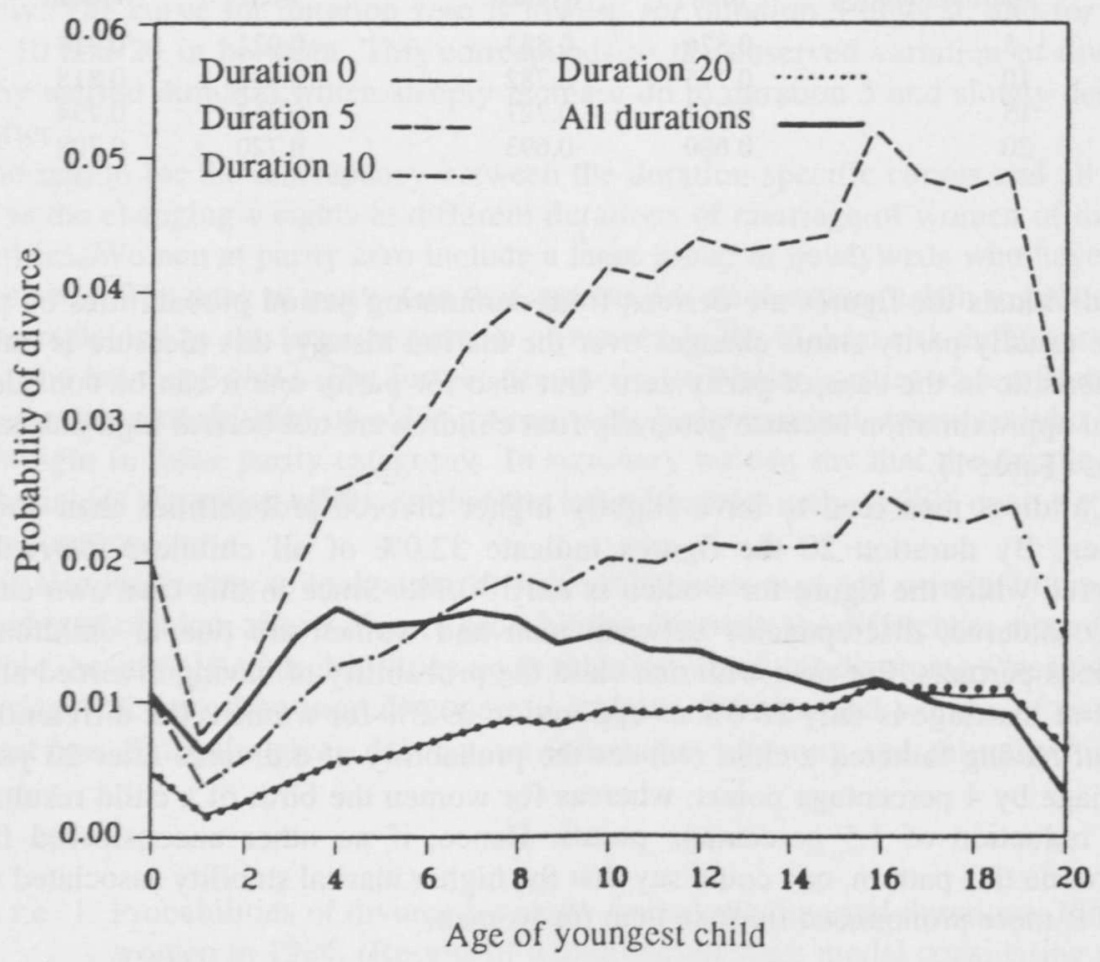

times higher than those with a child around age 2. Several interpretations of this phenomenon are possible. The presence of a baby could mean a common task making the relationship more important, or divorces of non-functioning relationships are simply postponed until the child is a little older.

\section{Effects of marital status on parity-specific birth probabilities}

The second half of this paper is devoted to the way in which divorce affects birth probabilities. Since we have no longitudinal data to study real birth or marriage cohorts, a comparison of parity-specific birth probabilities by age for different marital statuses can give us a first impression about the relevance of a divorce for childbearing behavior.

Figures $3 \mathrm{a}$ to $3 \mathrm{c}$ give the empirically-measured age-specific birth probabilities for single, married, and divorced women by parity. When interpreting the figures for married and especially for divorced women at young age groups we have to be aware of the fact that these are very selective groups. A married woman at age 20 has an annual probability of having a first birth of about 0.4 . For a divorced woman of the same age the birth probability is about half of that, whereas for single women it is about one-tenth. But even for the mid to late twenties when the number of divorced women is already sizable, fertility rates for divorced parity zero women are significantly higher than those for unmarried women and about one-third to one-fourth of that of married women. At higher ages the difference diminishes further. 
Fig u r e 3a-c. Birth probabilities by age and marital status for women with parities zero (a), one (b), and two (c). (Empirically-derived probabilities for 1984.)

a)

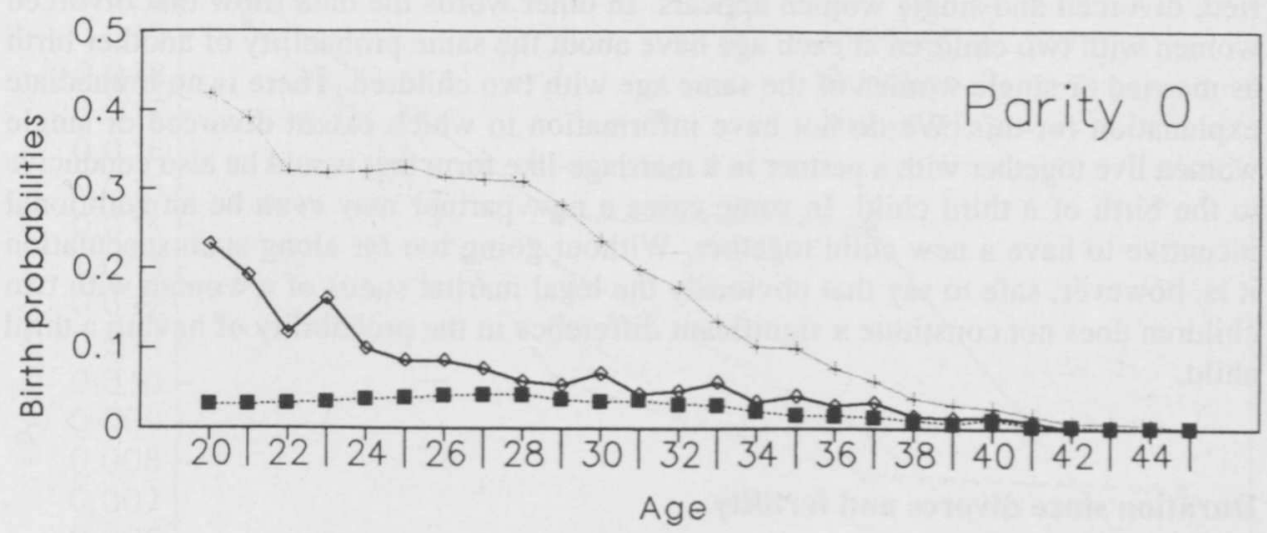

b)

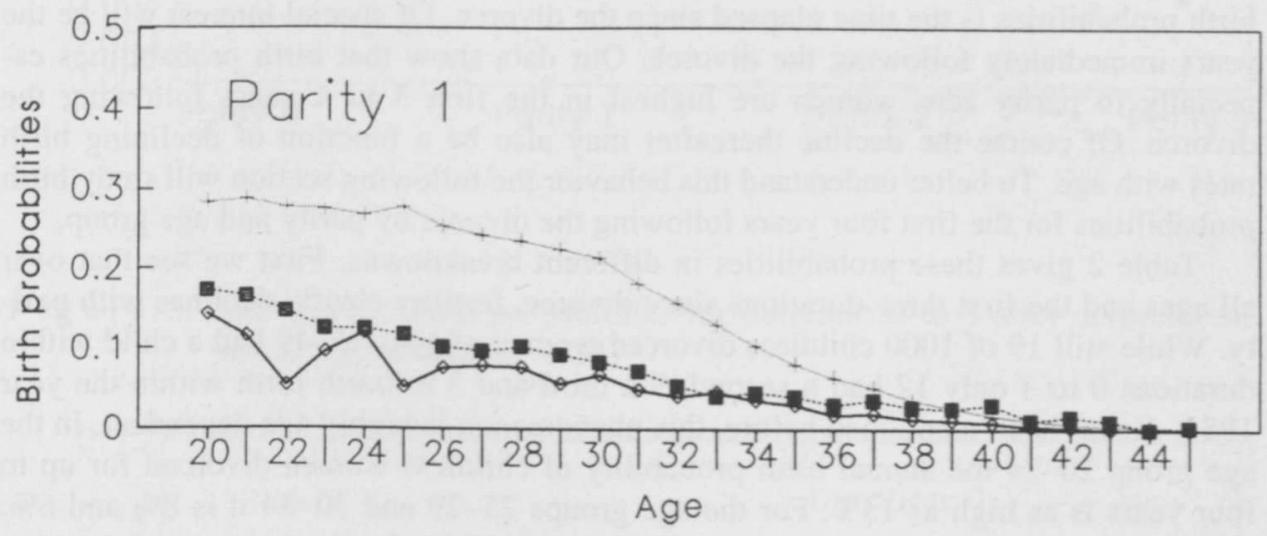

c)

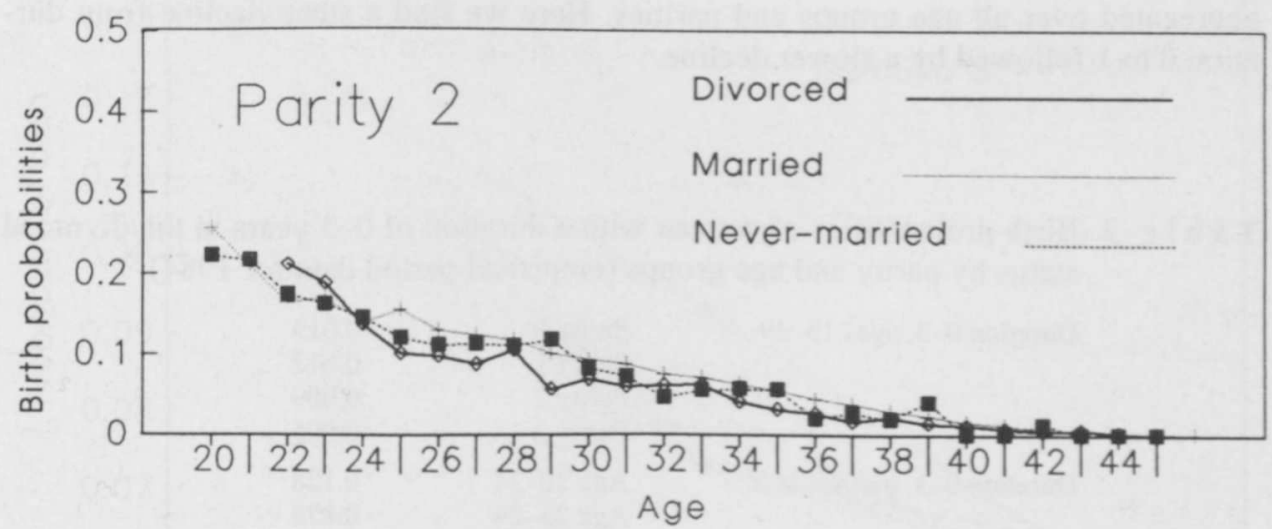


For second births, i.e. births to parity one women, the fertility of divorced women is clearly below that of single women. In the lower twenties the fertility of single women is at about half the level of married women while divorced women show a rather irregular pattern at a still lower level.

For third births the very interesting pattern of no clear differences between married, divorced and single women appears. In other words the data show that divorced women with two children at each age have about the same probability of another birth as married or single women of the same age with two children. There is no immediate explanation for this. We do not have information to which extent divorced or single women live together with a partner in a marriage-like form that would be also conducive to the birth of a third child. In some cases a new partner may even be an additional incentive to have a new child together. Without going too far along such speculation it is, however, safe to say that obviously the legal marital status of a woman with two children does not constitute a significant difference in the probability of having a third child.

\section{Duration since divorce and fertility}

One additional piece of information to shed more light on the effect of divorce on birth probabilities is the time elapsed since the divorce. Of special interest will be the years immediately following the divorce. Our data show that birth probabilities especially to parity zero women are highest in the first 3 to 4 years following the divorce. Of course the decline thereafter may also be a function of declining birth rates with age. To better understand this behavior the following section will study birth probabilities for the first four years following the divorce by parity and age group.

Table 2 gives these probabilities in different breakdowns. First we see that over all ages and the first three durations since divorce, fertility clearly declines with parity. While still 19 of 1000 childless divorced women of ages 15-49 had a child within durations 0 to 3 only 12 had a second, 9 a third and 5 a fourth birth within the year 1984. As has been mentioned before, this phenomenon is highly age dependent. In the age group 20-24 the annual birth probability of childless women divorced for up to four years is as high as $13 \%$. For the age groups $25-29$ and $30-34$ it is $8 \%$ and $6 \%$, respectively. Table 2 finally gives the birth probabilities by single years of duration aggregated over all age groups and parities. Here we find a steep decline from duration 0 to 1 followed by a slower decline.

$\mathrm{T}$ a b l e 2. Birth probabilities of women with a duration of $0-3$ years in the divorced status by parity and age groups (empirical period data for 1984).

$\begin{array}{lll}\text { Duration 0-3, ages 15-49 } & \text { Parity 0 } & 0.019 \\ & \text { Parity 1 } & 0.012 \\ & \text { Parity 2 } & 0.009 \\ & \text { Parity 3 } & 0.005 \\ \text { Duration 0-3, parities 0-3 } & \text { Age 20-24 } & 0.128 \\ & \text { Age 25-29 } & 0.078 \\ & \text { Age 30-34 } & 0.056 \\ \text { Parities 0-3, ages 15-49 } & \text { Duration 0 } & 0.014 \\ & \text { Duration 1 } & 0.011 \\ & \text { Duration 2 } & 0.010 \\ & \text { Duration 3 } & 0.010\end{array}$


F i g u r e 4. Parity-specific birth probabilities for durations 0 to 3 after divorce (empirical period data for 1984)

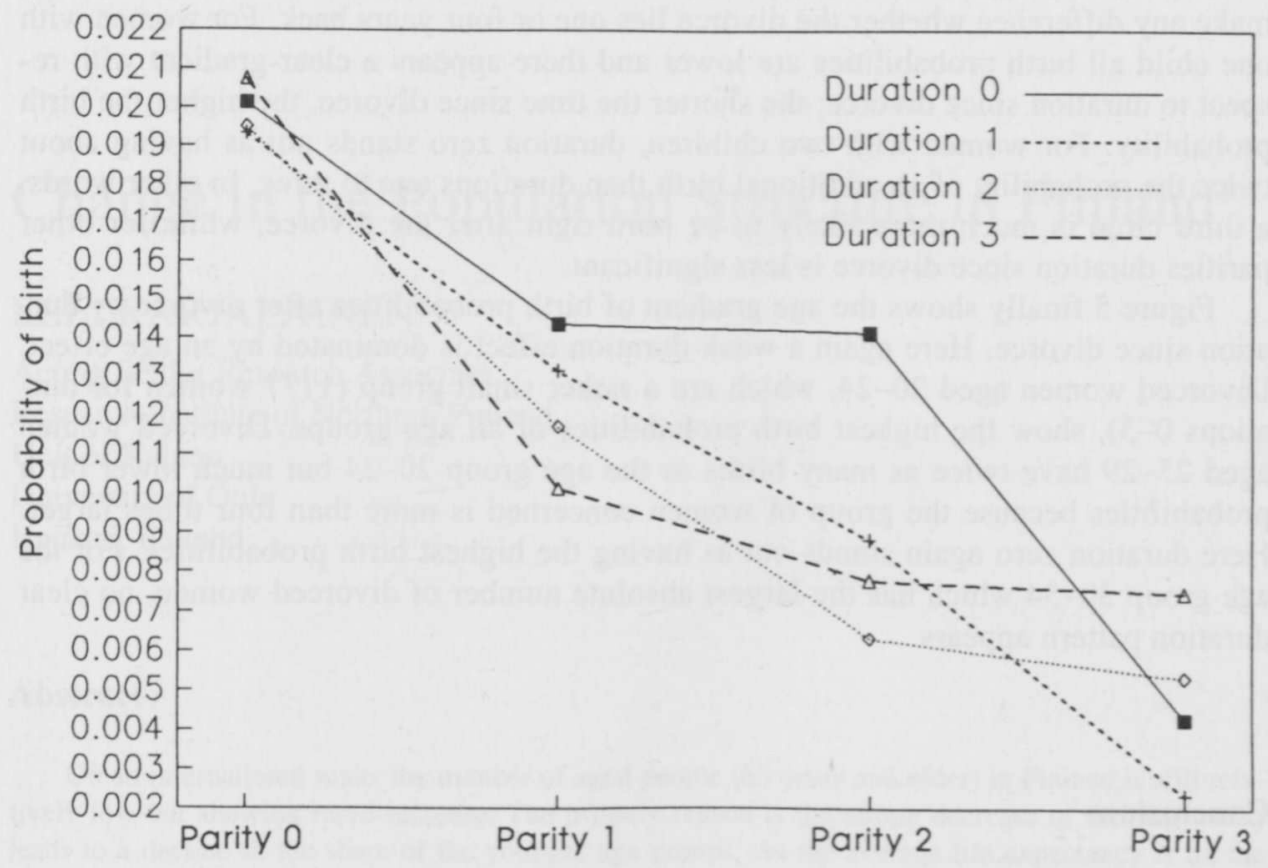

Fig u re 5. Age-specific birth probabilities for durations 0 to 3 after divorce (empirical period data for 1984)

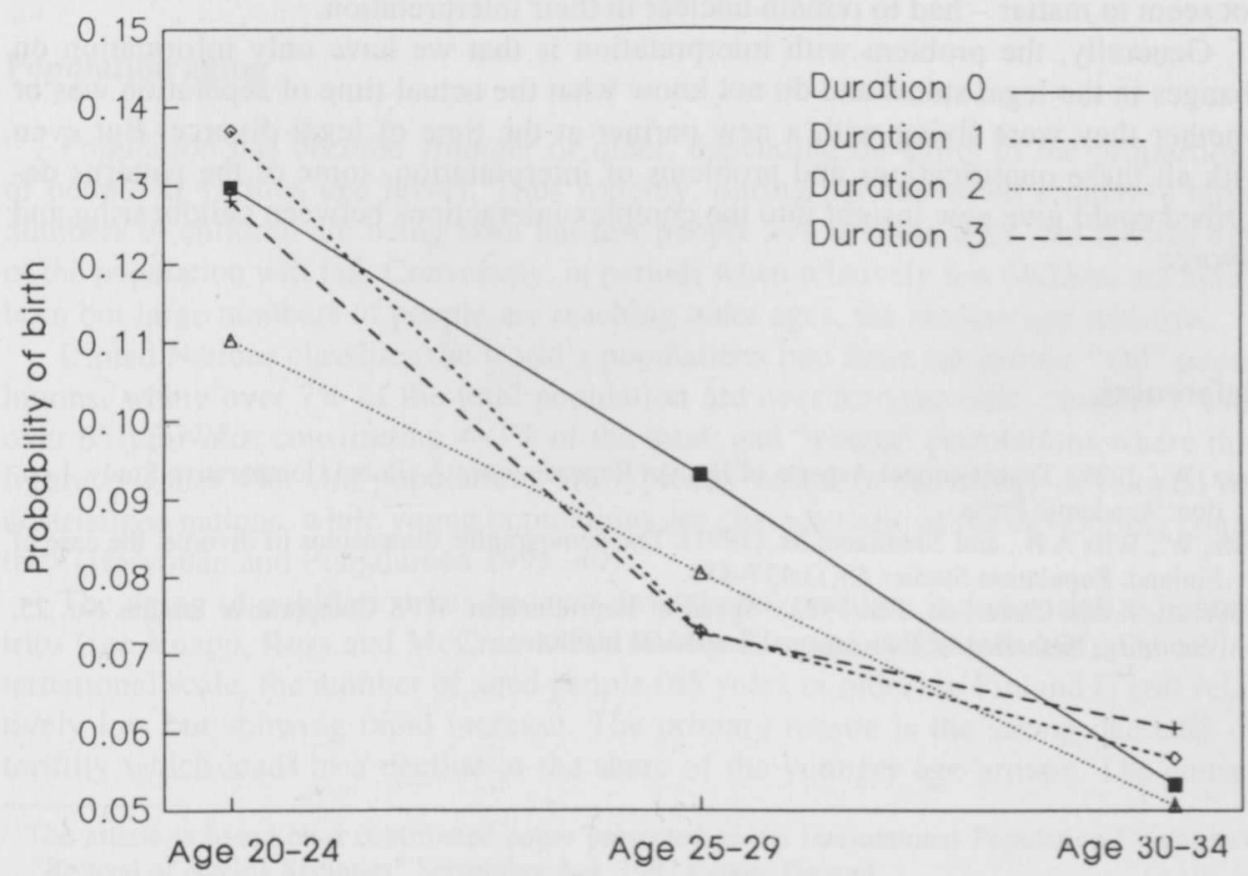


Figures 4 and 5 give more detail about this phenomenon. Figure 4 indicates that the decline of birth probabilities for divorced women along parity is much more significant than along duration since divorce. For childless women it actually does not make any difference whether the divorce lies one or four years back. For women with one child all birth probabilities are lower and there appears a clear gradient with respect to duration since divorce: the shorter the time since divorce, the higher the birth probability. For women with two children, duration zero stands out as having about twice the probability of an additional birth than durations one to three. In other words, a third child is much more likely to be born right after the divorce, while for other parities duration since divorce is less significant.

Figure 5 finally shows the age gradient of birth probabilities after divorce by duration since divorce. Here again a weak duration effect is dominated by an age effect. Divorced women aged 20-24, which are a rather small group (1177 women for durations 0-3), show the highest birth probabilities of all age groups. Divorced women aged 25-29 have twice as many births as the age group 20-24 but much lower birth probabilities because the group of women concerned is more than four times larger. Here duration zero again stands out as having the highest birth probabilities. For the age group 30-34 which has the largest absolute number of divorced women, no clear duration pattern appears.

\section{Conclusion}

This paper has described some demographic patterns that appear in the interrelation between divorce and fertility. Some of the appearing patterns were expected; others were surprising to the author. Of the surprising ones some - such as the difference between the patterns of divorce probabilities by parity (Figure 1) for specific marital durations and all durations together - could be sufficiently explained; others - such as the finding that for third birth probabilities, the marital status of the woman does not seem to matter - had to remain unclear in their interpretation.

Generally, the problem with interpretation is that we have only information on changes in the legal status and do not know what the actual time of separation was or whether they were living with a new partner at the time of legal divorce. But even with all these qualifications and problems of interpretation, some of the patterns described could give new insight into the complex interactions between childbearing and divorce.

\section{References}

Lutz, W.( 1989). Distributional Aspects of Human Reproduction: A Global Comparative Study. London: Academic Press.

Lutz, W., Wils A.B., and Nieminen, M. (1991). The demographic dimensions of divorce: the case of Finland. Population Studies 45(1):437-453.

Hobcraft, J. and Casterline, J.B.(1983). Speed of Reproduction. WFS Comparative Studies No. 25. Voorburg, Netherlands: International Statistical Institute. 